Article

\title{
Synthesis, In Vitro $\alpha$-Glucosidase Inhibitory Activity and Molecular Docking Studies of Novel Benzothiazole-Triazole Derivatives
}

\author{
Zipeng Gong ${ }^{1,2,3}$, Yaping Peng ${ }^{4}$, Jie Qiu ${ }^{4}$, Anbai Cao ${ }^{4}$, Guangcheng Wang ${ }^{4, *}$ and \\ Zhiyun Peng ${ }^{4, *}$ \\ 1 Provincial Key Laboratory of Pharmaceutics in Guizhou Province, Guizhou Medical University, \\ Beijing Road, Guiyang 550004, China; gzp4012607@126.com \\ 2 School of Pharmacy, Guizhou Medical University, 4 Beijing Road, Guiyang 550004, China \\ 3 National Engineering Research Center of Miao's Medicines, 4 Beijing Road, Guiyang 550004, China \\ 4 College of Chemistry and Chemical Engineering, Hunan Engineering Laboratory for Analyse and Drugs \\ Development of Ethnomedicine in Wuling Mountains, Jishou University, Jishou 416000, China; \\ yapingpeng17@163.com (Y.P.); qiujie_jsu@126.com (J.Q.); AnbaiCao1997@163.com (A.C.) \\ * Correspondence: wanggch123@163.com or wanggch123@jsu.edu.cn (G.W.); pengzhiyun1986@163.com or \\ pzy2017@jsu.edu.cn (Z.P.); Tel.: +86-743-856-3911
}

Received: 29 August 2017; Accepted: 13 September 2017; Published: 15 September 2017

\begin{abstract}
Benzothiazole-triazole derivatives $\mathbf{6 a}-\mathbf{6 s}$ have been synthesized and characterized by ${ }^{1} \mathrm{H}$-NMR and ${ }^{13} \mathrm{C}$-NMR. All synthetic compounds were screened for their in vitro $\alpha$-glucosidase inhibitory activity by using Baker's yeast $\alpha$-glucosidase enzyme. The majority of compounds exhibited a varying degree of $\alpha$-glucosidase inhibitory activity with $\mathrm{IC}_{50}$ values between 20.7 and $61.1 \mu \mathrm{M}$ when compared with standard acarbose $\left(\mathrm{IC}_{50}=817.38 \mu \mathrm{M}\right)$. Among the series, compound $6 \mathbf{s}$ $\left(\mathrm{IC}_{50}=20.7 \mu \mathrm{M}\right)$ bearing a chlorine group at the 5-position of the benzothiazole ring and a tert-butyl group at the para position of the phenyl ring, was found to be the most active compound. Preliminary structure-activity relationships were established. Molecular docking studies were performed to predict the binding interaction of the compounds in the binding pocket of the enzyme.
\end{abstract}

Keywords: benzothiazole; triazole; $\alpha$-glucosidase; molecular docking

\section{Introduction}

Type 2 diabetes is the most common type of diabetes, accounting for about 95 percent of diabetes cases. It is characterized by high blood glucose (hyperglycemia), insulin resistance and relative lack of insulin [1]. Hyperglycemia plays an important role in the development of type 2 diabetes and complications associated with diseases such as microvascular and macrovascular diseases [2]. $\alpha$-Glucosidase catalyzes the hydrolysis of terminal 1,4-linked $\alpha$-D-glucose residues and releases free $\alpha$-D-glucose, which is to the main cause of hyperglycemia [3]. Inhibition of $\alpha$-glucosidase activity is one of the important therapeutic approaches for the treatment of type 2 diabetes by slowing the absorption process of glucose in intestine [4]. Three inhibitors of $\alpha$-glucosidase enzyme (acarbose, miglitol, and voglibose) are being clinically used for the management of type 2 diabetes, and these are also used as anticancer [5], anti-HIV [6], and anti-hepatitis agents [7].

Benzothiazole is an important scaffold in bioorganic and medicinal chemistry, and has broad applications in drug discovery and development [8]. Benzothiazole-based compounds exhibit numerous biological activities such as anticonvulsant [9], anti-inflammatory [10], antimicrobial [11], anticancer [12] and anti-diabetic activities [13]. Some of benzothiazole-containing drugs, such as riluzole (anticonvulsant), ethoxzolamide (glaucoma, ulcers and as a diuretic), and zopolrestat (diabetic nephropathy and diabetes), have been used clinically for many years (Figure 1). Recently, M. Taha et al. 
reported the synthesis of a new class of benzothiazole hybrid with benzohydrazide moiety, and some of these showed superior activity compared to standard acarbose [14].<smiles>CCOc1ccc2nc(S(N)(=O)=O)sc2c1</smiles>

ethoxzolamide<smiles>C[C@]1(Cn2ccnn2)[C@H](C(=O)O)N2C(=O)C[C@H]2S1(=O)=O</smiles>

tazobactam<smiles>Nc1nc2ccc(OC(F)(F)F)cc2s1</smiles>

riluzole<smiles>CCOC(=O)OCc1nc2ccc(C(F)(F)F)cc2s1</smiles><smiles>NC(=O)c1nnn(Cc2cc(Cl)c(C(=O)c3ccc(Cl)cc3)c(Cl)c2)c1N</smiles>

carboxyamidotriazole

Figure 1. The structures of some commercial drugs containing benzothiazole or 1,2,3-triazole moiety.

On the other hand, 1,2,3-triazoles are an important class of heterocyclic compounds, and have been widely applied in various fields, including synthetic organic chemistry, biological science, material science and medicinal chemistry [15,16]. Notably, 1,2,3-triazole derivatives have been reported to exhibit various biological activities such as antioxidant [17], antibacterial [18], antitubercular [19] and anticancer [20]. There are some clinical and commercial drugs, such as tazobactam, cefatrizine and carboxyamidotriazole, with 1,2,3-triazole moiety (Figure 1). In particular, several recent studies have shown that some 1,2,3-triazole derivatives exhibit $\alpha$-glucosidase inhibitory activity [21-25].

In continuation of our drug discovery research on potential antidiabetic agents [25-28], we synthesized a novel series of benzothiazole-triazole derivatives and evaluated for their in vitro $\alpha$-glucosidase inhibitory activity. Furthermore, molecular docking was also performed to predict the binding interaction of the compounds in the binding pocket of the enzyme.

\section{Results and Discussion}

\subsection{Chemistry}

The synthesis of benzothiazole-triazole derivatives $\mathbf{6 a - 6 s}$ is shown in Scheme 1. The reaction of various benzyl chlorides or benzyl bromides 1 with $\mathrm{NaN}_{3}$ in DMF at room temperature for $24 \mathrm{~h}$ provided the corresponding (azidomethyl)aryl 2. Treatment of substituted 2-aminobenzenethiol with $\mathrm{CS}_{2}$ in the presence of $\mathrm{KOH}$ in reflux $\mathrm{EtOH} / \mathrm{H}_{2} \mathrm{O}$ afforded substituted benzo[d]thiazole-2-thiol 4, which reacted with 3-bromoprop-1-yne to provide the key intermediate 5 . In the final step, according to Huisgen, the 1,3-dipolar cycloaddition reaction (click reaction), condensation of intermediate 5 with various (azidomethyl)aryl 2 in the presence of sodium ascorbate and $\mathrm{CuSO}_{4} \cdot 5 \mathrm{H}_{2} \mathrm{O}$ in DMF solution resulted in the formation of the title products $6 \mathbf{a}-\mathbf{6 s}$.

The structures of all the title compounds $6 \mathbf{6}-\mathbf{6 s}$ were characterized by ${ }^{1} \mathrm{H}-\mathrm{NMR}$ and ${ }^{13} \mathrm{C}-\mathrm{NMR}$ spectra (Supplementary Materials). For instance, the ${ }^{1} \mathrm{H}-\mathrm{NMR}$ spectrum of $6 \mathrm{a}$ shows two singlet signals at $\delta 4.68 \mathrm{ppm}$ and $\delta 5.55 \mathrm{ppm}$, which were assigned to the methylene protons of $\mathrm{SCH}_{2}$ and $\mathrm{NCH}_{2}$, respectively. A doublet of two protons appeared at $\delta 7.19 \mathrm{ppm}(J=8.4 \mathrm{~Hz})$, which was assigned to the aromatic protons of $\mathrm{C} 2,6-\mathrm{H}$ of the right phenyl ring. The benzothiazole protons appeared as two doublets of one proton each at $\delta 7.86$ and $\delta 7.99 \mathrm{ppm}$, and a triplet of one proton at $\delta 7.37 \mathrm{ppm}$ with a coupling constant of $8.0 \mathrm{~Hz}$ and $8.4 \mathrm{~Hz}$, respectively. The rest of the protons of benzothiazole and the right phenyl appeared as a multiplet of three protons between $\delta 7.45$ and $7.51 \mathrm{ppm}$. The single 
peak of the C-H of triazole ring was observed at $\delta 8.17 \mathrm{ppm}$. The ${ }^{13} \mathrm{C}-\mathrm{NMR}$ spectrum of 6 a showed two characteristic peaks for methylene carbon at $\delta 27.9 \mathrm{ppm}$ and $\delta 52.5 \mathrm{ppm}$. The remaining thirteen signals were assigned to benzothiazole, triazole and phenyl carbon in the compound $6 \mathbf{a}$. Therefore, the data of ${ }^{1} \mathrm{H}-\mathrm{NMR}$ and ${ }^{13} \mathrm{C}-\mathrm{NMR}$ is in agreement with the structure of compound $\mathbf{6 a}$.

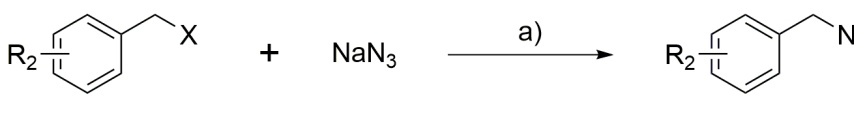

1: $\mathrm{X}=\mathrm{Cl}$ or $\mathrm{Br}$<smiles>[R]c1ccc(S)c(N)c1</smiles><smiles>[R]c1ccc2sc(SCC#C)nc2c1</smiles>

5
2

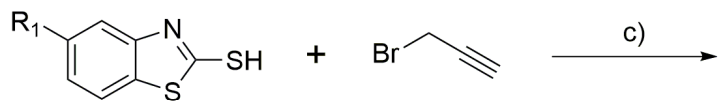

4

reflux, $5 \mathrm{~h}$; (d) sodium ascorbate, $\mathrm{CuSO}_{4} \cdot 5 \mathrm{H}_{2} \mathrm{O}$, DMF, r.t., $4 \mathrm{~h}$.

\section{2. $\alpha$-Glucosidase Inhibition Assay}

All the synthetic benzothiazole-triazole derivatives (6a-6s) were screened to evaluate their in vitro $\alpha$-glucosidase inhibitory activity by using Baker's yeast $\alpha$-glucosidase enzyme. The results are summarized in Table 1. The majority of compounds exhibited a varying degree of $\alpha$-glucosidase inhibitory activity with $\mathrm{IC}_{50}$ values between 20.7 and $61.1 \mu \mathrm{M}$ when compared with standard acarbose $\left(\mathrm{IC}_{50}=817.38 \mu \mathrm{M}\right)$. Among the series, compounds $6 \mathbf{a}, \mathbf{6 e}, \mathbf{6 i}, \mathbf{6 m}, \mathbf{6 n}, \mathbf{6 0}$ and $\mathbf{6 s}$ displayed potent inhibitory activity with $\mathrm{IC}_{50}$ values of $28.7,27.4,29.4,28.2,28.0,22.3$ and $20.7 \mu \mathrm{M}$.

Table 1. $\alpha$-Glucosidase inhibitory activity of novel benzothiazole-triazole derivatives (6a-6s).

\begin{tabular}{cccc}
\hline Compound & $\mathbf{R}_{\mathbf{1}}$ & $\mathbf{R}_{\mathbf{2}}$ & $\mathbf{I C}_{\mathbf{5 0}}(\boldsymbol{\mu} \mathbf{M})$ \\
\hline $\mathbf{6 a}$ & $\mathrm{H}$ & $4-\mathrm{Br}$ & 28.7 \\
$\mathbf{6 b}$ & $\mathrm{H}$ & $2-\mathrm{Cl}$ & $>100$ \\
$\mathbf{6 c}$ & $\mathrm{H}$ & $2-\mathrm{Br}$ & 37.4 \\
$\mathbf{6 d}$ & $\mathrm{H}$ & $3-\mathrm{F}$ & 61.1 \\
$\mathbf{6 e}$ & $\mathrm{H}$ & $4-\mathrm{Cl}$ & 27.4 \\
$\mathbf{6 f}$ & $\mathrm{H}$ & $2-\mathrm{F}$ & $>100$ \\
$\mathbf{6 g}$ & $\mathrm{H}$ & $\mathrm{H}$ & $>100$ \\
$\mathbf{6 h}$ & $\mathrm{H}$ & $3,5-\mathrm{Cl}$ & 41.0 \\
$\mathbf{6 i}$ & $\mathrm{H}$ & $4-\mathrm{tBu}$ & 29.4 \\
$\mathbf{6 j}$ & $\mathrm{Cl}$ & $2-\mathrm{F}$ & 45.9 \\
$\mathbf{6 k}$ & $\mathrm{Cl}$ & $2-\mathrm{Br}$ & 48.4 \\
$\mathbf{6 1}$ & $\mathrm{Cl}$ & $2-\mathrm{Cl}$ & 33.6 \\
$\mathbf{6 m}$ & $\mathrm{Cl}$ & $4-\mathrm{Cl}$ & 28.2 \\
$\mathbf{6} \mathbf{n}$ & $\mathrm{Cl}$ & $4-\mathrm{Br}$ & 28.0 \\
$\mathbf{6 0}$ & $\mathrm{Cl}$ & $3,5-\mathrm{Cl} 2$ & 22.3 \\
$\mathbf{6} \mathbf{p}$ & $\mathrm{Cl}$ & $3-\mathrm{F}$ & 38.6 \\
$\mathbf{6 q}$ & $\mathrm{Cl}$ & $4-\mathrm{F}$ & 53.1 \\
$\mathbf{6 r}$ & $\mathrm{Cl}$ & $\mathrm{H}$ & 44.4 \\
$\mathbf{6 s}$ & $\mathrm{Cl}$ & $4-\mathrm{tBu}$ & 20.7 \\
Acarbose & & & 817.38 \\
\hline
\end{tabular}




\subsection{Structure-Activity Relationship}

The structure-activity relationship of this class of compounds has been summarized. The introduction of electron withdrawing groups such as fluorine, chlorine, and bromine into the phenyl ring results in an increase in inhibitory activity. Furthermore, shifting these groups from the para to the ortho position decreased inhibitory activity. Introduction of the electron-withdrawing group chlorine into the benzothiazole ring results in a significant increase in inhibitory activity. It is interesting to point out that $6 \mathbf{i}$ and $\mathbf{6 s}$ containing the tert-butyl group at the para position of the phenyl ring exhibited potent $\alpha$-glucosidase inhibitory activity, with $\mathrm{IC}_{50}$ values of 29.4 and $20.7 \mu \mathrm{M}$, respectively. In particular, compound $6 \mathrm{~s}$ with a chlorine group at the 5-position of the benzothiazole ring and a tert-butyl group at the para position of the phenyl ring, was found to be the most active compound in this series. In order to illustrate the binding interactions of molecules into the active site of $\alpha$-glucosidase, molecular docking studies were conducted.

\subsection{Molecular Docking}

The theoretical binding mode between $6 \mathbf{i}$ and Saccharomyces cerevisiae $\alpha$-glucosidase is shown in Figure 2. Compound 6i adopted an "L-shaped" conformation in the pocket of the $\alpha$-glucosidase. The benzothiazole group of $\mathbf{6} \mathbf{i}$ was located at the hydrophobic pocket, surrounded by the residues Phe-157, Phe-310 and Phe-311, while the 4-(tert-butyl)phenyl group of $6 \mathbf{i}$ stretched into the hydrophobic pocket consisting of Phe-157, Phe-177 and Phe-300, forming a stable hydrophobic binding. Detailed analysis showed that the triazole group in the middle of $\mathbf{6} \mathbf{i}$ formed $\mathrm{CH}-\pi$ interaction with the residue Phe-300. In addition, cation- $\pi$ interactions were observed between $6 \mathbf{i}$ and the residues Lys-155, Arg-312 and Arg-439. Furthermore, 6i formed anion- $\pi$ interactions with the residues Glu-276 and Asp-349, respectively. All these interactions helped $6 \mathbf{i}$ to anchor in the binding site of the $\alpha$-glucosidase.

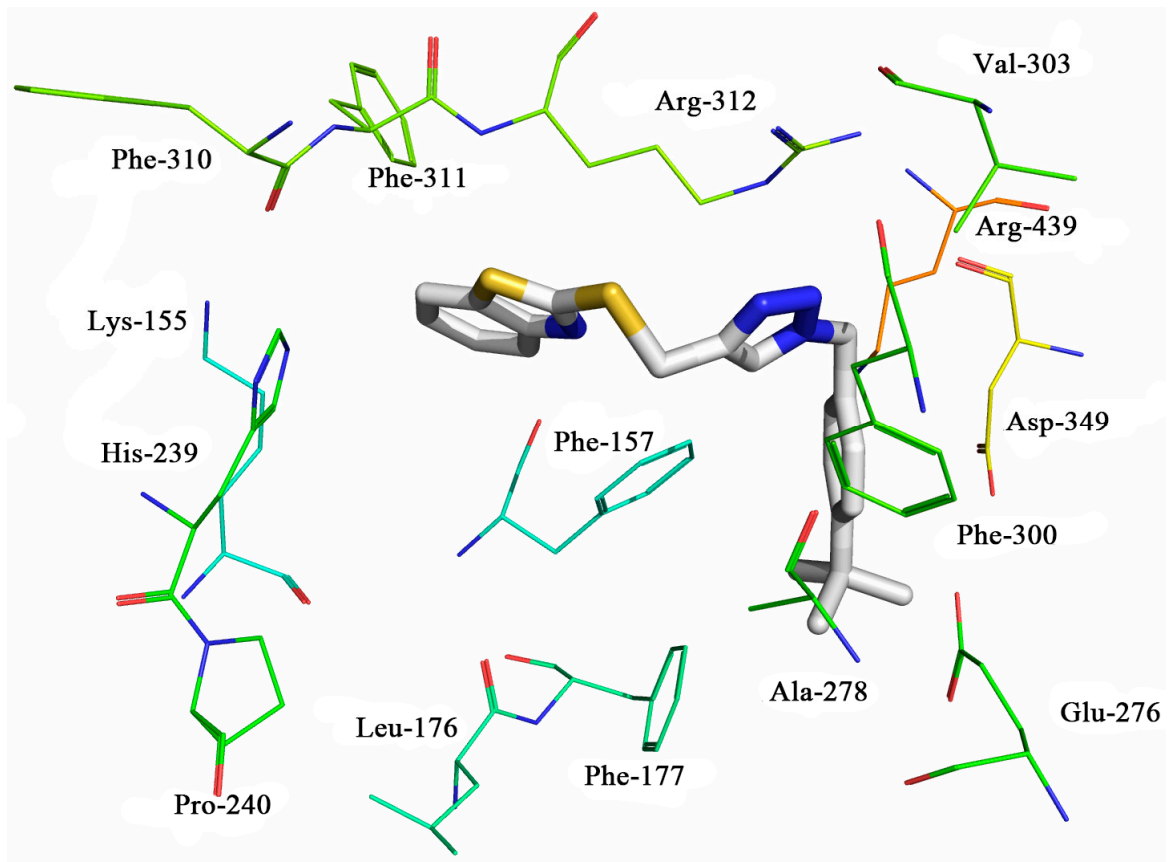

Figure 2. Compound $\mathbf{6 i}$ was docked to the binding pocket of the Saccharomyces cerevisiae $\alpha$-glucosidase.

To explain the activity order of $6 \mathrm{i}$ and $6 \mathrm{~s}$ against $\alpha$-glucosidase, $6 \mathrm{~s}$ was then docked to the binding site of $\alpha$-glucosidase; the theoretical binding mode between $6 \mathrm{~s}$ and $\alpha$-glucosidase is shown in Figure 3A. The interaction between $6 \mathbf{s}$ and $\alpha$-glucosidase was almost the same as the precursor 6i. The only difference was that the benzothiazole group of $6 \mathbf{s}$ formed $\pi-\pi$ stacking interactions with the residues His-239 and Phe-157, which were not formed by $\mathbf{6 i}$, making $\mathbf{6 s}$ more active than $\mathbf{6} \mathbf{i}$ 
against $\alpha$-glucosidase (Figure 3B). In addition, the estimated binding energies were $-8.6 \mathrm{kcal} \cdot \mathrm{mol}^{-1}$ for $6 \mathbf{i}$ and $-8.9 \mathrm{kcal} \cdot \mathrm{mol}^{-1}$ for $6 \mathrm{~s}$, respectively, which was consistent with the results of the in vitro anti- $\alpha$-glucosidase assay. In summary, the above molecular simulations give us rational explanation of the interactions between $\mathbf{6} \mathbf{i}, \mathbf{6 s}$ and $\alpha$-glucosidase, which provided valuable information for further development of $\alpha$-glucosidase inhibitors.

A

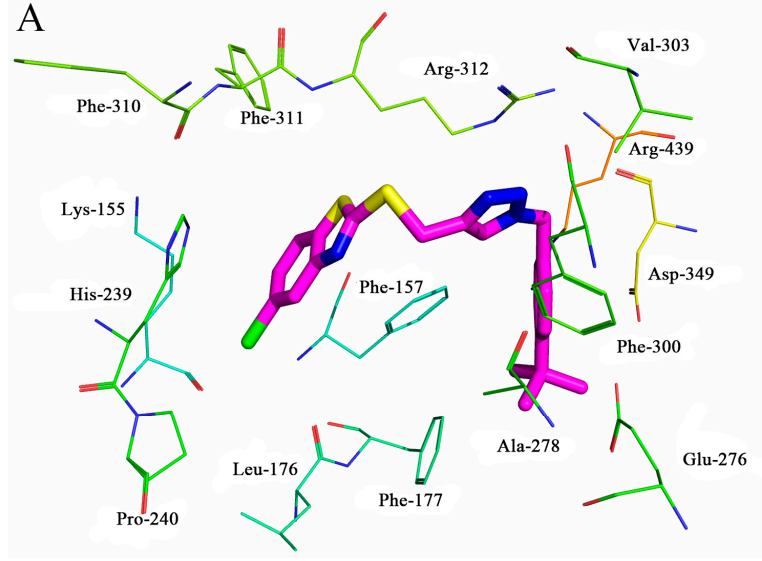

B

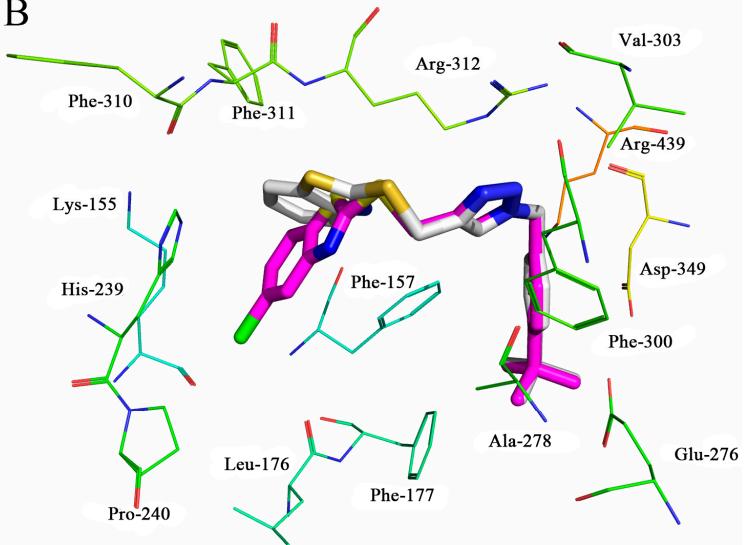

Figure 3. (A) Compound $6 \mathrm{~s}$ was docked to the binding pocket of the Saccharomyces cerevisiae $\alpha$-glucosidase; (B) Compounds $6 \mathbf{i}$ and 6 s were docked to the binding pocket of the Saccharomyces cerevisiae $\alpha$-glucosidase (overlapped).

\section{Experimental Section}

\subsection{General}

All starting materials and reagents were purchased from commercial suppliers. Nuclear magnetic resonance spectra (NMR) were recorded on a Bruker spectrometer (400 MHz) with TMS as an external reference and reported in parts per million.

\subsection{General Procedure for the Synthesis of $\mathbf{2}$}

A mixture of substituted benzyl chlorides or benzyl bromides $1(1.0 \mathrm{mmol})$ and $\mathrm{NaN}_{3}(1.2 \mathrm{mmol})$ in $10 \mathrm{~mL}$ DMF was stirred at room temperature for $24 \mathrm{~h}$. After the completion of the reaction (monitored by TLC), the mixture was poured into water and extracted 3 times for ethyl acetate. The combined organic layers were dried over $\mathrm{Na}_{2} \mathrm{SO}_{4}$ and concentrated under vacuum. The residue was purified by chromatography to give 2 .

\subsection{General Procedure for the Synthesis of $\mathbf{4}$}

To a solution of substituted 2-aminobenzenethiol $(2 \mathrm{mmol})$ in EtOH $(20 \mathrm{~mL})$ was added $\mathrm{CS}_{2}$ $(4 \mathrm{mmol})$ and $\mathrm{KOH}(4 \mathrm{mmol})$, and the mixture was stirred at $80{ }^{\circ} \mathrm{C}$ for $12 \mathrm{~h}$. After cooling to the room temperature, the mixture was treated with ice-water $(100 \mathrm{~mL})$ and $10 \% \mathrm{HCl}$ was added to adjust $\mathrm{pH}$ to $2-3$. The solid precipitate was collected by filtration to obtain 4 , which was used in the next step without further purification.

\subsection{General Procedure for the Synthesis of $\mathbf{5}$}

A mixture of 4 (1 mmol), 3-bromoprop-1-yne $(1.5 \mathrm{mmol})$ and $\mathrm{K}_{2} \mathrm{CO}_{3}(3 \mathrm{mmol})$ in acetone $(20 \mathrm{~mL})$ was stirred at reflux for $5 \mathrm{~h}$. The solvent was evaporated under reduced pressure and the residue was purified by chromatography to give 5 . 


\subsection{General Procedure for the Synthesis of Benzothiazole-Triazole Derivatives (6a-6s)}

A mixture of $5(1.0 \mathrm{mmol}), 2(1.0 \mathrm{mmol}), \mathrm{CuSO}_{4} \cdot 5 \mathrm{H}_{2} \mathrm{O}(0.025 \mathrm{~g} ; 0.1 \mathrm{mmol})$ and sodium ascorbate $(0.10 \mathrm{~g}, 0.5 \mathrm{mmol})$ in DMF $(10 \mathrm{~mL})$ was stirred at room temperature for $4 \mathrm{~h}$. After completion of reaction, the content was poured into $50 \mathrm{~mL}$ ice cold water and extracted with ethylacetate $(50 \mathrm{~mL} \times 3)$. The combined organic extracts were dried over $\mathrm{Na}_{2} \mathrm{SO}_{4}$, filtered, and concentrated. The residue was purified by chromatography on silica gel with EtOAc/petroleum ether to give the title products 6a-6s.

2-(((1-(4-Bromobenzyl)-1H-1,2,3-triazol-4-yl)methyl)thio)benzo[d] thiazole (6a). Yellow solid, yield 65\%, m.p. 112-114 ${ }^{\circ} \mathrm{C} .{ }^{1} \mathrm{H}-\mathrm{NMR}\left(d_{6}-\mathrm{DMSO}, 400 \mathrm{MHz}\right) \delta: 4.68\left(\mathrm{~s}, 2 \mathrm{H}, \mathrm{SCH}_{2}\right), 5.55\left(\mathrm{~s}, 2 \mathrm{H}, \mathrm{NCH}_{2}\right), 7.19(\mathrm{~d}, 2 \mathrm{H}$, $J=8.4 \mathrm{~Hz}, \mathrm{ArH}), 7.37(\mathrm{t}, 1 \mathrm{H}, J=8.4 \mathrm{~Hz}, \mathrm{ArH}), 7.45-7.51(\mathrm{~m}, 3 \mathrm{H}, \mathrm{ArH}), 7.86(\mathrm{~d}, 1 \mathrm{H}, J=8.0 \mathrm{~Hz}, \mathrm{ArH})$, $7.99(\mathrm{~d}, 1 \mathrm{H}, J=8.0 \mathrm{~Hz}, \mathrm{ArH}), 8.17$ (s, 1H, CH-triazole); ${ }^{13} \mathrm{C}-\mathrm{NMR}\left(d_{6}\right.$-DMSO, $\left.100 \mathrm{MHz}\right) \delta: 27.9,52.5$, 121.7, 121.8, 122.3, 124.6, 125.0, 126.8, 130.5, 132.1, 135.2, 135.8, 143.2, 153.0, 166.1; MS (ESI, m/z): 416.96 $[\mathrm{M}+\mathrm{H}]^{+}$.

2-(((1-(2-Chlorobenzyl)-1H-1,2,3-triazol-4-yl)methyl)thio)benzo[d]thiazole (6b). Yellow solid, yield 79\%, m.p. $110-111^{\circ} \mathrm{C} .{ }^{1} \mathrm{H}-\mathrm{NMR}\left(d_{6}-\mathrm{DMSO}, 400 \mathrm{MHz}\right) \delta: 4.69\left(\mathrm{~s}, 2 \mathrm{H}, \mathrm{SCH}_{2}\right), 5.66\left(\mathrm{~s}, 2 \mathrm{H}, \mathrm{NCH}_{2}\right), 7.11(\mathrm{dd}$, $1 \mathrm{H}, J=8.0 \mathrm{~Hz}, 1.2 \mathrm{~Hz}, \mathrm{ArH}), 7.27(\mathrm{dt}, 1 \mathrm{H}, J=8.0 \mathrm{~Hz}, 1.2 \mathrm{~Hz}, \mathrm{ArH}), 7.33-7.39$ (m, 2H, ArH), 7.45-7.49 $(\mathrm{m}, 2 \mathrm{H}, \mathrm{ArH}), 7.86(\mathrm{~d}, 1 \mathrm{H}, J=8.0 \mathrm{~Hz}, \mathrm{ArH}), 7.99$ (d, 1H, J = 8.0 Hz, ArH), 8.15 (s, 1H, CH-triazole); ${ }^{13} \mathrm{C}-\mathrm{NMR}\left(d_{6}\right.$-DMSO, $\left.100 \mathrm{MHz}\right) \delta: 27.9,51.1,121.7,122.3,125.0,125.0,126.8,128.1,130.0,130.6,130.7$, 133.0, 133.7, 135.2, 143.0, 153.0, 166.1; MS (ESI, $m / z): 373.03[\mathrm{M}+\mathrm{H}]^{+}$.

2-(((1-(2-Bromobenzyl)-1H-1,2,3-triazol-4-yl)methyl)thio)benzo[d]thiazole (6c). Yellow solid, yield 52\%, m.p. 104-106 ${ }^{\circ} \mathrm{C} .{ }^{1} \mathrm{H}-\mathrm{NMR}\left(d_{6}\right.$-DMSO, $\left.400 \mathrm{MHz}\right) \delta: 4.70\left(\mathrm{~s}, 2 \mathrm{H}, \mathrm{SCH}_{2}\right), 5.64\left(\mathrm{~s}, 2 \mathrm{H}, \mathrm{NCH}_{2}\right), 7.05(\mathrm{dd}, 1 \mathrm{H}$, $J=8.0 \mathrm{~Hz}, 1.2 \mathrm{~Hz}, \mathrm{ArH}), 7.24-7.33(\mathrm{~m}, 2 \mathrm{H}, \mathrm{ArH}), 7.37(\mathrm{dt}, 1 \mathrm{H}, J=8.0 \mathrm{~Hz}, 1.2 \mathrm{~Hz}, \mathrm{ArH}), 7.47(\mathrm{dt}, 1 \mathrm{H}$, $J=8.0 \mathrm{~Hz}, 1.2 \mathrm{~Hz}, \mathrm{ArH}), 7.62(\mathrm{dd}, 1 \mathrm{H}, J=8.0 \mathrm{~Hz}, 1.2 \mathrm{~Hz}, \mathrm{ArH}), 7.86(\mathrm{~d}, 1 \mathrm{H}, J=8.0 \mathrm{~Hz}, \mathrm{ArH}), 7.99$ (d, $1 \mathrm{H}, J=8.0 \mathrm{~Hz}, \mathrm{ArH}), 8.14$ (s, 1H, CH-triazole); ${ }^{13} \mathrm{C}-\mathrm{NMR}\left(d_{6}-\mathrm{DMSO}, 100 \mathrm{MHz}\right) \delta: 27.9,53.4,121.7$, 122.3, 123.2, 125.0, 125.0, 126.8, 128.6, 130.7, 130.8, 133.3, 135.2, 135.3, 143.0, 153.0, 166.0; MS (ESI, $m / z)$ : $416.98[\mathrm{M}+\mathrm{H}]^{+}$.

2-(((1-(3-Fluorobenzyl)-1H-1,2,3-triazol-4-yl)methyl)thio)benzo[d]thiazole (6d). White solid, yield 67\%, m.p. 112-114 ${ }^{\circ} \mathrm{C} .{ }^{1} \mathrm{H}-\mathrm{NMR}\left(d_{6}\right.$-DMSO, $\left.400 \mathrm{MHz}\right) \delta: 4.69\left(\mathrm{~s}, 2 \mathrm{H}, \mathrm{SCH}_{2}\right), 5.59\left(\mathrm{~s}, 2 \mathrm{H}, \mathrm{NCH}_{2}\right), 7.06-7.16(\mathrm{~m}, 3 \mathrm{H}$, ArH), 7.33-7.38 (m, 2H, ArH), 7.47 (dt, 1H, J = 8.0 Hz, 1.2 Hz, ArH), 7.87 (d, 1H, J = 8.0 Hz, ArH), 7.99 $(\mathrm{d}, 1 \mathrm{H}, J=8.0 \mathrm{~Hz}, \mathrm{ArH}), 8.22$ (s, 1H, CH-triazole); ${ }^{13} \mathrm{C}-\mathrm{NMR}\left(d_{6}\right.$-DMSO, $\left.100 \mathrm{MHz}\right) \delta: 27.9,52.6,115.1$ $(\mathrm{d}, 1 \mathrm{C}, J=21.0 \mathrm{~Hz}), 115.3(\mathrm{~d}, 1 \mathrm{C}, J=21.0 \mathrm{~Hz}), 121.7,122.3,124.3(\mathrm{~d}, 1 \mathrm{C}, J=2.8 \mathrm{~Hz}), 124.7,125.0,126.8$, $131.2(\mathrm{~d}, 1 \mathrm{C}, J=8.3 \mathrm{~Hz}), 135.2,139.1$ (d, 1C, $J=7.5 \mathrm{~Hz}), 143.2,153.0,161.3$ (d, 1C, J = 243 Hz), 166.1; MS $(\mathrm{ESI}, \mathrm{m} / z): 357.06[\mathrm{M}+\mathrm{H}]^{+}$.

2-(((1-(4-Chlorobenzyl)-1H-1,2,3-triazol-4-yl)methyl)thio)benzo[d]thiazole (6e). White solid, yield 81\%, m.p. 119-120 ${ }^{\circ} \mathrm{C} .{ }^{1} \mathrm{H}-\mathrm{NMR}\left(d_{6}-\mathrm{DMSO}, 400 \mathrm{MHz}\right) \delta: 4.68\left(\mathrm{~s}, 2 \mathrm{H}, \mathrm{SCH}_{2}\right), 5.57\left(\mathrm{~s}, 2 \mathrm{H}, \mathrm{NCH}_{2}\right), 7.25(\mathrm{~d}, 2 \mathrm{H}$, $J=8.4 \mathrm{~Hz}, \mathrm{ArH}), 7.36(\mathrm{~d}, 2 \mathrm{H}, J=8.4 \mathrm{~Hz}, \mathrm{ArH}), 7.40(\mathrm{dd}, 1 \mathrm{H}, J=8.4 \mathrm{~Hz}, 2.0 \mathrm{~Hz}, \mathrm{ArH}), 7.96(\mathrm{~d}, 1 \mathrm{H}$, $J=2.0 \mathrm{~Hz}, \mathrm{ArH}), 8.03(\mathrm{~d}, 1 \mathrm{H}, J=8.4 \mathrm{~Hz}, \mathrm{ArH}), 8.20$ (s, 1H, CH-triazole); ${ }^{13} \mathrm{C}-\mathrm{NMR}\left(d_{6}\right.$-DMSO, 100 $\mathrm{MHz}) \delta:$ 28.0, 52.5, 121.2, 123.7, 124.8, 125.0, 129.1, 130.2, 131.7, 133.3 134.0, 135.4, 143.0, 153.9, 169.0; MS (ESI, $m / z): 373.03[\mathrm{M}+\mathrm{H}]^{+}$.

2-(((1-(2-Fluorobenzyl)-1H-1,2,3-triazol-4-yl)methyl)thio)benzo[d]thiazole (6f). Yellow solid, yield 64\%, m.p. 86-88 ${ }^{\circ} \mathrm{C} .{ }^{1} \mathrm{H}-\mathrm{NMR}\left(d_{6}-\mathrm{DMSO}, 400 \mathrm{MHz}\right) \delta: 4.69\left(\mathrm{~s}, 2 \mathrm{H}, \mathrm{SCH}_{2}\right), 5.63\left(\mathrm{~s}, 2 \mathrm{H}, \mathrm{NCH}_{2}\right), 7.12-7.28(\mathrm{~m}, 3 \mathrm{H}$, ArH), 7.35-7.39 (m, 2H, ArH), 7.47 (dt, 1H, J = 8.0 Hz, 1.2 Hz, ArH), 7.86 (d, 1H, J = 8.0 Hz, ArH), 7.99 $(\mathrm{d}, 1 \mathrm{H}, J=8.0 \mathrm{~Hz}, \mathrm{ArH}), 8.16\left(\mathrm{~s}, 1 \mathrm{H}, \mathrm{CH}\right.$-triazole); ${ }^{13} \mathrm{C}-\mathrm{NMR}\left(d_{6}\right.$-DMSO, $\left.100 \mathrm{MHz}\right) \delta: 27.9,47.3(\mathrm{~d}, 1 \mathrm{C}$, $J=3.8 \mathrm{~Hz}), 115.9(\mathrm{~d}, 1 \mathrm{C}, J=20.8 \mathrm{~Hz}), 121.7,122.3,123.2(\mathrm{~d}, 1 \mathrm{C}, J=14.6 \mathrm{~Hz}), 124.7,125.0,125.2(\mathrm{~d}, 1 \mathrm{C}$, $J=3.5 \mathrm{~Hz}), 126.8,131.0(\mathrm{~d}, 1 \mathrm{C}, J=3.5 \mathrm{~Hz}), 131.1(\mathrm{~d}, 1 \mathrm{C}, J=8.2 \mathrm{~Hz}), 135.2,143.1,153.0,159.3(\mathrm{~d}, 1 \mathrm{C}$, $J=245 \mathrm{~Hz}), 166.1$; MS (ESI, $m / z): 357.06[\mathrm{M}+\mathrm{H}]^{+}$. 
2-(((1-Benzyl-1H-1,2,3-triazol-4-yl)methyl)thio)benzo[d]thiazole (6g) [29]. White solid, yield 72\%, m.p. 109-110 ${ }^{\circ} \mathrm{C} .{ }^{1} \mathrm{H}-\mathrm{NMR}\left(d_{6}\right.$-DMSO, $\left.400 \mathrm{MHz}\right) \delta: 4.68\left(\mathrm{~s}, 2 \mathrm{H}, \mathrm{SCH}_{2}\right), 5.56\left(\mathrm{~s}, 2 \mathrm{H}, \mathrm{NCH}_{2}\right), 7.23-7.25(\mathrm{~m}, 2 \mathrm{H}$, ArH), 7.28-7.31 (m, 3H, ArH), $7.37(\mathrm{dt}, 1 \mathrm{H}, J=8.0 \mathrm{~Hz}, 1.2 \mathrm{~Hz}, \mathrm{ArH}), 7.47(\mathrm{dt}, 1 \mathrm{H}, J=8.0 \mathrm{~Hz}, 1.2 \mathrm{~Hz}$, $\mathrm{ArH}), 7.87(\mathrm{~d}, 1 \mathrm{H}, J=8.0 \mathrm{~Hz}, \mathrm{ArH}), 8.00(\mathrm{~d}, 1 \mathrm{H}, J=8.0 \mathrm{~Hz}, \mathrm{ArH}), 8.18(\mathrm{~s}, 1 \mathrm{H}, \mathrm{CH}$-triazole $) ;{ }^{13} \mathrm{C}-\mathrm{NMR}$ $\left(d_{6}\right.$-DMSO, $\left.100 \mathrm{MHz}\right) \delta: 27.9,53.2,121.7,122.3,124.6,125.0,126.8,128.3,128.5,129.2,135.2,136.4,143.1$, 153.1, 166.1; MS (ESI, $m / z): 339.07[\mathrm{M}+\mathrm{H}]^{+}$.

2-(((1-(3,5-Dichlorobenzyl)-1H-1,2,3-triazol-4-yl)methyl)thio)benzo[d]thiazole (6h). White solid, yield 75\%, m.p. 85-87 ${ }^{\circ} \mathrm{C} .{ }^{1} \mathrm{H}-\mathrm{NMR}\left(d_{6}\right.$-DMSO, $\left.400 \mathrm{MHz}\right) \delta: 4.69\left(\mathrm{~s}, 2 \mathrm{H}, \mathrm{SCH}_{2}\right), 5.65\left(\mathrm{~s}, 2 \mathrm{H}, \mathrm{NCH}_{2}\right), 7.15(\mathrm{~d}, 1 \mathrm{H}$, $J=8.0 \mathrm{~Hz}, \mathrm{ArH}), 7.35-7.39(\mathrm{~m}, 2 \mathrm{H}, \mathrm{ArH}), 7.47(\mathrm{dt}, 1 \mathrm{H}, J=8.0 \mathrm{~Hz}, 1.2 \mathrm{~Hz}, \mathrm{ArH}), 7.64(\mathrm{~d}, 1 \mathrm{H}, J=2.0 \mathrm{~Hz}$, $\mathrm{ArH}), 7.86(\mathrm{~d}, 1 \mathrm{H}, J=8.0 \mathrm{~Hz}, \mathrm{ArH}), 8.00(\mathrm{~d}, 1 \mathrm{H}, J=8.0 \mathrm{~Hz}, \mathrm{ArH}), 8.15(\mathrm{~s}, 1 \mathrm{H}, \mathrm{CH}$-triazole $) ;{ }^{13} \mathrm{C}-\mathrm{NMR}$ $\left(d_{6}\right.$-DMSO, $\left.100 \mathrm{MHz}\right) \delta: 27.9,50.5,121.7,122.3,125.0,126.8,128.3,129.6,132.2,132.8,134.1,134.4,135.2$, 143.1, 153.0, 166.0; MS (ESI, $m / z): 406.99[\mathrm{M}+\mathrm{H}]^{+}$.

2-(((1-(4-(Tert-butyl)benzyl)-1H-1,2,3-triazol-4-yl)methyl)thio)benzo[d]thiazole (6i). Yellow solid, yield 51\%, m.p. $77-78{ }^{\circ} \mathrm{C} .{ }^{1} \mathrm{H}-\mathrm{NMR}\left(d_{6}\right.$-DMSO, $\left.400 \mathrm{MHz}\right) \delta: 1.22\left(\mathrm{~s}, 9 \mathrm{H}, \mathrm{CH}_{3}\right), 4.68\left(\mathrm{~s}, 2 \mathrm{H}, \mathrm{SCH}_{2}\right), 5.51(\mathrm{~s}, 2 \mathrm{H}$, $\left.\mathrm{NCH}_{2}\right), 7.15(\mathrm{~d}, 2 \mathrm{H}, J=8.4 \mathrm{~Hz}, \mathrm{ArH}), 7.29(\mathrm{~d}, 2 \mathrm{H}, J=8.4 \mathrm{~Hz}, \mathrm{ArH}), 7.37(\mathrm{t}, 1 \mathrm{H}, J=8.0 \mathrm{~Hz}, \mathrm{ArH})$, $7.47(\mathrm{t}, 1 \mathrm{H}, J=8.0 \mathrm{~Hz}, \mathrm{ArH}), 7.87(\mathrm{~d}, 1 \mathrm{H}, J=8.0 \mathrm{~Hz}, \mathrm{ArH}), 7.99(\mathrm{~d}, 1 \mathrm{H}, J=8.0 \mathrm{~Hz}, \mathrm{ArH}), 8.16(\mathrm{~s}, 1 \mathrm{H}$, CH-triazole); ${ }^{13} \mathrm{C}-\mathrm{NMR}\left(d_{6}\right.$-DMSO, $\left.100 \mathrm{MHz}\right) \delta: 27.9,31.5,34.7,53.0,121.7,122.3,124.5,125.0,125.9$, $126.8,128.1,133.5,135.2,143.1,151.0,153.1,166.1$; MS (ESI, $m / z): 395.13[\mathrm{M}+\mathrm{H}]^{+}$.

5-Chloro-2-(((1-(2-fluorobenzyl)-1H-1,2,3-triazol-4-yl)methyl)thio)benzo[d]thiazole (6j). White solid, yield 63\%, m.p. $115-117^{\circ} \mathrm{C} .{ }^{1} \mathrm{H}-\mathrm{NMR}\left(d_{6}\right.$-DMSO, $\left.400 \mathrm{MHz}\right) \delta: 4.68\left(\mathrm{~s}, 2 \mathrm{H}, \mathrm{SCH}_{2}\right), 5.63\left(\mathrm{~s}, 2 \mathrm{H}, \mathrm{NCH}_{2}\right)$, 7.12-7.27 (m, 3H, ArH), 7.35-7.40 (m, 1H, ArH), 7.40 (dd, 1H, J = 8.0 Hz, 2.0 Hz, ArH), $7.94(\mathrm{~d}, 1 \mathrm{H}$, $J=2.0 \mathrm{~Hz}, \mathrm{ArH}), 8.03(\mathrm{~d}, 1 \mathrm{H}, J=8.0 \mathrm{~Hz}, \mathrm{ArH}), 8.17(\mathrm{~s}, 1 \mathrm{H}, \mathrm{CH}$-triazole $) ;{ }^{13} \mathrm{C}-\mathrm{NMR}\left(d_{6}\right.$-DMSO, $\left.100 \mathrm{MHz}\right)$ $\delta: 28.0,47.3(\mathrm{~d}, 1 \mathrm{C}, J=3.9 \mathrm{~Hz}), 115.9(\mathrm{~d}, 1 \mathrm{C}, J=20.8 \mathrm{~Hz}), 121.2,123.2(\mathrm{~d}, 1 \mathrm{C}, J=14.6 \mathrm{~Hz}), 123.7,124.8$, 125.0, $125.2(\mathrm{~d}, 1 \mathrm{C}, J=3.6 \mathrm{~Hz}), 131.0(\mathrm{~d}, 1 \mathrm{C}, J=3.5 \mathrm{~Hz}), 131.9(\mathrm{~d}, 1 \mathrm{C}, J=8.2 \mathrm{~Hz}), 131.6,134.0,142.9$, 153.9, $159.2(\mathrm{~d}, 1 \mathrm{C}, J=245 \mathrm{~Hz}), 169.0 ; \mathrm{MS}(\mathrm{ESI}, \mathrm{m} / z): 391.02[\mathrm{M}+\mathrm{H}]^{+}$.

2-(((1-(2-Bromobenzyl)-1H-1,2,3-triazol-4-yl)methyl)thio)-5-chlorobenzo[d]thiazole (6k). White solid, yield 79\%, m.p. $119-120{ }^{\circ} \mathrm{C} .{ }^{1} \mathrm{H}-\mathrm{NMR}\left(d_{6}\right.$-DMSO, $\left.400 \mathrm{MHz}\right) \delta: 4.69\left(\mathrm{~s}, 2 \mathrm{H}, \mathrm{SCH}_{2}\right), 5.64\left(\mathrm{~s}, 2 \mathrm{H}, \mathrm{NCH}_{2}\right), 7.05$ $(\mathrm{dd}, 1 \mathrm{H}, J=8.0 \mathrm{~Hz}, 2.0 \mathrm{~Hz}, \mathrm{ArH}), 7.24-7.33(\mathrm{~m}, 2 \mathrm{H}, \mathrm{ArH}), 7.40(\mathrm{dd}, 1 \mathrm{H}, J=8.0 \mathrm{~Hz}, 2.0 \mathrm{~Hz}, \mathrm{ArH}), 7.61$ $(\mathrm{dd}, 1 \mathrm{H}, J=8.0 \mathrm{~Hz}, 1.2 \mathrm{~Hz}, \mathrm{ArH}), 7.93(\mathrm{~d}, 1 \mathrm{H}, J=2.0 \mathrm{~Hz}, \mathrm{ArH}), 8.02(\mathrm{~d}, 1 \mathrm{H}, J=8.8 \mathrm{~Hz}, \mathrm{ArH}), 8.16(\mathrm{~s}, 1 \mathrm{H}$, CH-triazole); ${ }^{13} \mathrm{C}-\mathrm{NMR}\left(d_{6}\right.$-DMSO, $\left.100 \mathrm{MHz}\right) \delta: 28.0,53.4,121.2,123.2,123.7,125.0,125.2,128.6,130.7$, $130.8,131.6,133.3,134.0,135.3,142.8,153.9,168.9$; MS (ESI, $m / z): 450.93[\mathrm{M}+\mathrm{H}]^{+}$.

5-Chloro-2-(((1-(2-chlorobenzyl)-1H-1,2,3-triazol-4-yl)methyl)thio)benzo[d] thiazole (61). White solid, yield 66\%, m.p. ${ }^{128-130}{ }^{\circ} \mathrm{C} .{ }^{1} \mathrm{H}-\mathrm{NMR}\left(d_{6}\right.$-DMSO, $\left.400 \mathrm{MHz}\right) \delta: 4.69\left(\mathrm{~s}, 2 \mathrm{H}, \mathrm{SCH}_{2}\right), 5.66\left(\mathrm{~s}, 2 \mathrm{H}, \mathrm{NCH}_{2}\right), 7.11$ $(\mathrm{d}, 1 \mathrm{H}, J=8.0 \mathrm{~Hz}, \mathrm{ArH}), 7.27(\mathrm{t}, 1 \mathrm{H}, J=8.0 \mathrm{~Hz}, \mathrm{ArH}), 7.36(\mathrm{dt}, 1 \mathrm{H}, J=8.0 \mathrm{~Hz}, 2.0 \mathrm{~Hz}, \mathrm{ArH}), 7.40(\mathrm{dd}$, $1 \mathrm{H}, J=8.8 \mathrm{~Hz}, 2.0 \mathrm{~Hz}, \mathrm{ArH}), 7.45(\mathrm{~d}, 1 \mathrm{H}, J=8.0 \mathrm{~Hz}, \mathrm{ArH}), 7.94(\mathrm{~d}, 1 \mathrm{H}, J=2.0 \mathrm{~Hz}, \mathrm{ArH}), 8.03(\mathrm{~d}, 1 \mathrm{H}$, $J=8.8 \mathrm{~Hz}, \mathrm{ArH}), 8.16(\mathrm{~s}, 1 \mathrm{H}, \mathrm{CH}$-triazole $) ;{ }^{13} \mathrm{C}-\mathrm{NMR}\left(d_{6}-\mathrm{DMSO}, 100 \mathrm{MHz}\right) \delta: 28.0,51.1,121.2,123.7$, 125.0, 125.2, 128.1, 130.0, 130.6, 130.7, 131.6, 133.0, 133.7, 134.0, 142.8, 153.9, 168.9; MS (ESI, $m / z): 406.99$ $[\mathrm{M}+\mathrm{H}]^{+}$.

5-Chloro-2-(((1-(4-chlorobenzyl)-1H-1,2,3-triazol-4-yl)methyl)thio)benzo[d]thiazole (6m). White solid, yield 78\%, m.p. $111-112{ }^{\circ} \mathrm{C} .{ }^{1} \mathrm{H}-\mathrm{NMR}\left(d_{6}\right.$-DMSO, $\left.400 \mathrm{MHz}\right) \delta: 4.68\left(\mathrm{~s}, 2 \mathrm{H}, \mathrm{SCH}_{2}\right), 5.57\left(\mathrm{~s}, 2 \mathrm{H}, \mathrm{NCH}_{2}\right), 7.25$ $(\mathrm{d}, 2 \mathrm{H}, J=8.0 \mathrm{~Hz}, \mathrm{ArH}), 7.36(\mathrm{~d}, 2 \mathrm{H}, J=8.0 \mathrm{~Hz}, \mathrm{ArH}), 7.40(\mathrm{dd}, 1 \mathrm{H}, J=8.8 \mathrm{~Hz}, 2.0 \mathrm{~Hz}, \mathrm{ArH}), 7.95$ $(\mathrm{d}, 1 \mathrm{H}, J=2.0 \mathrm{~Hz}, \mathrm{ArH}), 8.02(\mathrm{~d}, 1 \mathrm{H}, J=8.4 \mathrm{~Hz}, \mathrm{ArH}), 8.20(\mathrm{~s}, 1 \mathrm{H}, \mathrm{CH}$-triazole $) ;{ }^{13} \mathrm{C}-\mathrm{NMR}\left(d_{6}\right.$-DMSO, $100 \mathrm{MHz}$ ) $\delta:$ 28.0, 52.4, 121.2, 123.7, 124.8, 125.0, 129.1, 130.2, 131.6, 133.3, 134.0, 135.4, 143.1, 153.9, 169.0; MS (ESI, $m / z): 406.99[\mathrm{M}+\mathrm{H}]^{+}$. 
2-(((1-(4-Bromobenzyl)-1H-1,2,3-triazol-4-yl)methyl)thio)-5-chlorobenzo[d]thiazole (6n). White solid, yield 73\%, m.p. $114-116{ }^{\circ} \mathrm{C} .{ }^{1} \mathrm{H}-\mathrm{NMR}\left(d_{6}\right.$-DMSO, $\left.400 \mathrm{MHz}\right) \delta: 4.68\left(\mathrm{~s}, 2 \mathrm{H}, \mathrm{SCH}_{2}\right), 5.55\left(\mathrm{~s}, 2 \mathrm{H}, \mathrm{NCH}_{2}\right), 7.19$ $(\mathrm{d}, 2 \mathrm{H}, J=8.4 \mathrm{~Hz}, \mathrm{ArH}), 7.41(\mathrm{dd}, 1 \mathrm{H}, J=8.4 \mathrm{~Hz}, 2.0 \mathrm{~Hz}, \mathrm{ArH}), 7.49(\mathrm{~d}, 2 \mathrm{H}, J=8.4 \mathrm{~Hz}, \mathrm{ArH}), 7.96$ $(\mathrm{d}, 1 \mathrm{H}, J=2.0 \mathrm{~Hz}, \mathrm{ArH}), 8.03(\mathrm{~d}, 1 \mathrm{H}, J=8.4 \mathrm{~Hz}, \mathrm{ArH}), 8.21\left(\mathrm{~s}, 1 \mathrm{H}, \mathrm{CH}\right.$-triazole); ${ }^{13} \mathrm{C}-\mathrm{NMR}\left(d_{6}\right.$-DMSO, $100 \mathrm{MHz}$ ) $\delta:$ 28.0, 52.5, 121.2, 121.8, 123.7, 124.8, 125.0, 130.5, 131.6, 132.1, 134.0, 135.9, 1443.1, 153.9, 169.0; MS (ESI, $m / z): 452.94[\mathrm{M}+\mathrm{H}]^{+}$.

5-Chloro-2-(((1-(3,5-dichlorobenzyl)-1H-1,2,3-triazol-4-yl)methyl)thio)benzo[d]thiazole (6o). Yellow solid, yield 55\%, m.p. ${ }^{124-126}{ }^{\circ} \mathrm{C} .{ }^{1} \mathrm{H}-\mathrm{NMR}\left(d_{6}-\mathrm{DMSO}, 400 \mathrm{MHz}\right) \delta: 4.69\left(\mathrm{~s}, 2 \mathrm{H}, \mathrm{SCH}_{2}\right), 5.65\left(\mathrm{~s}, 2 \mathrm{H}, \mathrm{NCH}_{2}\right)$, $7.15(\mathrm{~d}, 1 \mathrm{H}, J=8.4 \mathrm{~Hz}, \mathrm{ArH}), 7.36(\mathrm{dd}, 1 \mathrm{H}, J=8.4 \mathrm{~Hz}, 2.0 \mathrm{~Hz}, \mathrm{ArH}), 7.41(\mathrm{dd}, 1 \mathrm{H}, J=8.4 \mathrm{~Hz}, 2.0 \mathrm{~Hz}$, ArH), $7.63(\mathrm{~d}, 1 \mathrm{H}, J=2.0 \mathrm{~Hz}, \mathrm{ArH}), 7.94(\mathrm{~d}, 1 \mathrm{H}, J=2.0 \mathrm{~Hz}, \mathrm{ArH}), 8.03(\mathrm{~d}, 1 \mathrm{H}, J=8.4 \mathrm{~Hz}, \mathrm{ArH}), 8.17(\mathrm{~s}$, $1 \mathrm{H}, \mathrm{CH}$-triazole); ${ }^{13} \mathrm{C}-\mathrm{NMR}\left(d_{6}\right.$-DMSO, $\left.100 \mathrm{MHz}\right) \delta: 27.9,50.5,121.2,123.7,125.0,125.2,128.2,129.5$, 131.6, 132.1, 132.9, 134.0, 134.1, 134.4, 142.9, 153.9, 168.9; MS (ESI, $m / z): 440.95[\mathrm{M}+\mathrm{H}]^{+}$.

5-Chloro-2-(((1-(3-fluorobenzyl)-1H-1,2,3-triazol-4-yl)methyl)thio)benzo[d]thiazole (6p). White solid, yield 74\%, m.p. $140-141{ }^{\circ} \mathrm{C} .{ }^{1} \mathrm{H}-\mathrm{NMR}\left(d_{6}\right.$-DMSO, $\left.400 \mathrm{MHz}\right) \delta: 4.68\left(\mathrm{~s}, 2 \mathrm{H}, \mathrm{SCH}_{2}\right), 5.59\left(\mathrm{~s}, 2 \mathrm{H}, \mathrm{NCH}_{2}\right)$, 7.06-7.14 (m, 3H, ArH), 7.34-7.36 (m, 1H, ArH), 7.40 (dd, 1H, J = 8.4 Hz, $2.0 \mathrm{~Hz}, \mathrm{ArH}), 7.95(\mathrm{~d}, 1 \mathrm{H}$, $J=2.0 \mathrm{~Hz}, \mathrm{ArH}), 8.02(\mathrm{~d}, 1 \mathrm{H}, J=8.4 \mathrm{~Hz}, \mathrm{ArH}), 8.23(\mathrm{~s}, 1 \mathrm{H}, \mathrm{CH}$-triazole $) ;{ }^{13} \mathrm{C}-\mathrm{NMR}\left(d_{6}\right.$-DMSO, $\left.100 \mathrm{MHz}\right)$ $\delta: 28.0,52.6,115.0(\mathrm{~d}, 1 \mathrm{C}, J=21.9 \mathrm{~Hz}), 115.3(\mathrm{~d}, 1 \mathrm{C}, J=20.8 \mathrm{~Hz}), 121.2,123.7,124.3(\mathrm{~d}, 1 \mathrm{C}, J=2.8 \mathrm{~Hz})$, $124.9,125.0,131.2(\mathrm{~d}, 1 \mathrm{C}, J=5.3 \mathrm{~Hz}), 131.7,134.0,139.1(\mathrm{~d}, 1 \mathrm{C}, J=7.5 \mathrm{~Hz}), 143.1,153.9,161.3(\mathrm{~d}, 1 \mathrm{C}$, $J=243 \mathrm{~Hz}), 169.0$; MS (ESI, $m / z): 391.02[\mathrm{M}+\mathrm{H}]^{+}$.

5-Chloro-2-(((1-(4-fluorobenzyl)-1H-1,2,3-triazol-4-yl)methyl)thio)benzo[d]thiazole (6q). Yellow solid, yield 68\%, m.p. $108-109{ }^{\circ} \mathrm{C} .{ }^{1} \mathrm{H}-\mathrm{NMR}\left(d_{6}\right.$-DMSO, $\left.400 \mathrm{MHz}\right) \delta: 4.68\left(\mathrm{~s}, 2 \mathrm{H}, \mathrm{SCH}_{2}\right), 5.55$ (s, 2H, NCH 2$), 7.14$ $(\mathrm{d}, 2 \mathrm{H}, J=8.8 \mathrm{~Hz}, \mathrm{ArH}), 7.31-7.34(\mathrm{~m}, 2 \mathrm{H}, \mathrm{ArH}), 7.41(\mathrm{dd}, 1 \mathrm{H}, J=8.4 \mathrm{~Hz}, 2.0 \mathrm{~Hz}, \mathrm{ArH}), 7.95(\mathrm{~d}, 1 \mathrm{H}$, $J=2.0 \mathrm{~Hz}, \mathrm{ArH}), 8.03(\mathrm{~d}, 1 \mathrm{H}, J=8.4 \mathrm{~Hz}, \mathrm{ArH}), 8.19\left(\mathrm{~s}, 1 \mathrm{H}, \mathrm{CH}\right.$-triazole); ${ }^{13} \mathrm{C}-\mathrm{NMR}\left(d_{6}\right.$-DMSO, $\left.100 \mathrm{MHz}\right)$ $\delta: 28.0,52.5,115.9(\mathrm{~d}, 1 \mathrm{C}, J=21.5 \mathrm{~Hz}), 121.2,123.7,124.6,125.0,130.6(\mathrm{~d}, 1 \mathrm{C}, J=8.3 \mathrm{~Hz}), 131.6,132.7(\mathrm{~d}$, $1 C, J=3.1 \mathrm{~Hz}), 134.0,143.0,153.9,161.1(\mathrm{~d}, 1 \mathrm{C}, J=243 \mathrm{~Hz}), 169.0$; MS (ESI, $m / z): 391.02[\mathrm{M}+\mathrm{H}]^{+}$.

2-(((1-Benzyl-1H-1,2,3-triazol-4-yl)methyl)thio)-5-chlorobenzo[d]thiazole (6r) [29]. White solid, yield 75\%, m.p. $142-144{ }^{\circ} \mathrm{C} .{ }^{1} \mathrm{H}-\mathrm{NMR}\left(d_{6}-\mathrm{DMSO}, 400 \mathrm{MHz}\right) \delta: 4.68\left(\mathrm{~s}, 2 \mathrm{H}, \mathrm{SCH}_{2}\right), 5.56\left(\mathrm{~s}, 2 \mathrm{H}, \mathrm{NCH}_{2}\right), 7.25-7.30$ $(\mathrm{m}, 5 \mathrm{H}, \mathrm{ArH}), 7.41(\mathrm{~d}, 1 \mathrm{H}, J=8.4 \mathrm{~Hz}, \mathrm{ArH}), 7.96(\mathrm{~d}, 1 \mathrm{H}, J=2.0 \mathrm{~Hz}, \mathrm{ArH}), 8.03(\mathrm{~d}, 1 \mathrm{H}, J=8.4 \mathrm{~Hz}, \mathrm{ArH})$, 8.19 (s, $1 \mathrm{H}, \mathrm{CH}$-triazole); ${ }^{13} \mathrm{C}-\mathrm{NMR}\left(d_{6}\right.$-DMSO, $\left.100 \mathrm{MHz}\right) \delta: 28.0,53.3,121.2,123.7,124.7,125.0,128.3$, 128.5, 129.1, 131.6, 134.0, 136.4, 142.9, 153.9, 169.0; MS (ESI, $m / z): 373.03[\mathrm{M}+\mathrm{H}]^{+}$.

2-(((1-(4-(Tert-butyl)benzyl)-1H-1,2,3-triazol-4-yl)methyl)thio)-5-chlorobenzo[d]thiazole (6s). Yellow solid, yield 78\%, m.p. $94-95{ }^{\circ} \mathrm{C} .{ }^{1} \mathrm{H}-\mathrm{NMR}\left(d_{6}\right.$-DMSO, $\left.400 \mathrm{MHz}\right) \delta: 1.22\left(\mathrm{~s}, 9 \mathrm{H}, \mathrm{CH}_{3}\right), 4.67\left(\mathrm{~s}, 2 \mathrm{H}, \mathrm{SCH}_{2}\right), 5.51$ $\left(\mathrm{s}, 2 \mathrm{H}, \mathrm{NCH}_{2}\right), 7.15(\mathrm{~d}, 2 \mathrm{H}, J=8.4 \mathrm{~Hz}, \operatorname{ArH}), 7.28(\mathrm{~d}, 2 \mathrm{H}, J=8.4 \mathrm{~Hz}, \mathrm{ArH}), 7.40(\mathrm{~d}, 1 \mathrm{H}, J=8.4 \mathrm{~Hz}$, $\mathrm{ArH}), 7.97(\mathrm{~d}, 1 \mathrm{H}, J=2.0 \mathrm{~Hz}, \mathrm{ArH}), 8.03(\mathrm{~d}, 1 \mathrm{H}, J=8.4 \mathrm{~Hz}, \mathrm{ArH}), 8.19\left(\mathrm{~s}, 1 \mathrm{H}, \mathrm{CH}\right.$-triazole); ${ }^{13} \mathrm{C}-\mathrm{NMR}$ $\left(d_{6}\right.$-DMSO, $\left.100 \mathrm{MHz}\right) \delta: 28.0,31.5,34.7,53.0,121.2,123.7,124.7,125.0,125.9,128.0,131.6,133.5,134.0$, 142.9, 151.0, 153.9, 169.0; MS (ESI, $m / z): 429.09[\mathrm{M}+\mathrm{H}]^{+}$.

\subsection{In Vitro Assay of $\alpha$-Glucosidase Inhibitory Activity}

$\alpha$-Glucosidase inhibitory activity was assayed by using $0.1 \mathrm{M}$ phosphate buffer $(\mathrm{pH} 6.8)$ at $37^{\circ} \mathrm{C}$. The enzyme $(0.1 \mathrm{U} / \mathrm{mL})$ in phosphate buffer saline was incubated with various concentrations of test compounds at $37^{\circ} \mathrm{C}$ for $15 \mathrm{~min}$. Then $1.25 \mathrm{mM} p$-nitrophenyl $\alpha$-D-glucopyranoside was added to the mixture as a substrate. After further incubation at $37^{\circ} \mathrm{C}$ for $30 \mathrm{~min}$. The absorbance was measured spectrophotometrically at $405 \mathrm{~nm}$. The sample solution was replaced by DMSO as a control. Acarbose was used as a positive control. 


\subsection{Molecular Docking}

Molecular docking studies were performed to investigate the binding mode between $\mathbf{6 i}, \mathbf{6 s}$ and $\alpha$-glucosidase using Autodock vina 1.1.2. The 3D structure of $\alpha$-glucosidase of Saccharomyces cerevisiae have been predicted using homology modeling in our previous report [25]. The 3D structure of the compounds were obtained by ChemBioDraw Ultra 14.0 and ChemBio3D Ultra 14.0 softwares. The AutoDockTools 1.5.6 package was employed to generate the docking input files. The search grid of $\alpha$-glucosidase was identified as center_x: -19.676 , center_y: -7.243 , and center_z: -21.469 , with dimensions size_x: 15, size_y: 15, and size_z: 15 . The value of exhaustiveness was set to 20 . For Vina docking, the default parameters were used if it was not mentioned. The best-scoring pose as judged by the Vina docking score was chosen and visually analyzed using PyMoL 1.7.6 software (http://www.pymol.org/).

\section{Conclusions}

In summary, a novel series of benzothiazole-triazole derivatives 6a-6s have been synthesized and evaluated for their $\alpha$-glucosidase inhibitory activity. The majority of compounds exhibited superior $\alpha$-glucosidase inhibitory activity in the range of $\mathrm{IC}_{50}=20.7$ and $61.1 \mu \mathrm{M}$ as compared to standard acarbose $\left(\mathrm{IC}_{50}=817.38 \mu \mathrm{M}\right)$. Molecular docking study was carried out to understand the molecular interaction of compounds with the active site of $\alpha$-glucosidase. This study has identified a novel series of lead compounds which can be used for the development of new $\alpha$-glucosidase inhibitors.

Supplementary Materials: Supplementary Materials are available online.

Acknowledgments: This work was supported by Key Technology R \& D Program of Guizhou Province ([2017]2845); Natural Science Foundation of Jishou University (15JD004); Joint Foundation of Guizhou Province Department of Science and Technology and Guizhou Medical University ([2016]7346); Jishou University for talent introduction; The Open Project Program of Hunan Engineering Laboratory for Analyse and Drugs Development of Ethnomedicine in Wuling Mountains.

Author Contributions: Guangcheng Wang designed and wrote the paper; Zipeng Gong, Yaping Peng, Jie Qiu, Anbai Cao, and Zhiyun Peng carried out the experiments. All authors have read and approved the final manuscript.

Conflicts of Interest: The authors confirm that this article content has no conflict of interest.

\section{References}

1. Ross, S.A.; Gulve, E.A.; Wang, M.H. Chemistry and biochemistry of type 2 diabetes. Chem. Rev. 2004, 104, 1255-1282. [CrossRef] [PubMed]

2. King, G.L.; Loeken, M.R. Hyperglycemia-induced oxidative stress in diabetic complications. Histochem. Cell Biol. 2004, 122, 333-338. [CrossRef] [PubMed]

3. Hirsh, A.J.; Yao, S.Y.M.; Young, J.D.; Cheeseman, C.I. Inhibition of glucose absorption in the rat jejunum: A novel action of alpha-D-glucosidase inhibitors. Gastroenterology 1997, 113, 205-211. [CrossRef]

4. Joshi, S.R.; Standl, E.; Tong, N.; Shah, P.; Kalra, S.; Rathod, R. Therapeutic potential of alpha-glucosidase inhibitors in type 2 diabetes mellitus: An evidence-based review. Expert Opin. Pharmacother. 2015, 16, 1959-1981. [CrossRef] [PubMed]

5. Pili, R.; Chang, J.; Partis, R.A.; Mueller, R.A.; Chrest, F.J.; Passaniti, A. The alpha-glucosidase I inhibitor castanospermine alters endothelial cell glycosylation, prevents angiogenesis, and inhibits tumor growth. Cancer Res. 1995, 55, 2920-2926. [PubMed]

6. $\quad$ Rawlings, A.J.; Lomas, H.; Pilling, A.W.; Lee, M.J.R.; Alonzi, D.S.; Rountree, J.S.S.; Jenkinson, S.F.; Fleet, G.W.J.; Dwek, R.A.; Jones, J.H.; et al. Synthesis and Biological Characterisation of Novel N-Alkyl-Deoxynojirimycin alpha-Glucosidase Inhibitors. ChemBioChem 2009, 10, 1101-1105. [CrossRef] [PubMed]

7. Zitzmann, N.; Mehta, A.S.; Carrouée, S.; Butters, T.D.; Platt, F.M.; McCauley, J.; Blumberg, B.S.; Dwek, R.A.; Block, T.M. Imino sugars inhibit the formation and secretion of bovine viral diarrhea virus, a pestivirus model of hepatitis $C$ virus: Implications for the development of broad spectrum anti-hepatitis virus agents. Proc. Natl. Acad. Sci. USA 1999, 96, 11878-11882. [CrossRef] [PubMed] 
8. Keri, R.S.; Patil, M.R.; Patil, S.A.; Budagumpi, S. A comprehensive review in current developments of benzothiazole-based molecules in medicinal chemistry. Eur. J. Med. Chem. 2015, 89, 207-251. [CrossRef] [PubMed]

9. Siddiqui, N.; Rana, A.; Khan, S.A.; Bhat, M.A.; Haque, S.E. Synthesis of benzothiazole semicarbazones as novel anticonvulsants-The role of hydrophobic domain. Bioorg. Med. Chem. Lett. 2007, 17, 4178-4182. [CrossRef] [PubMed]

10. Kharbanda, C.; Alam, M.S.; Hamid, H.; Javed, K.; Bano, S.; Dhulap, A.; Ali, Y.; Nazreen, S.; Haider, S. Synthesis and evaluation of pyrazolines bearing benzothiazole as anti-inflammatory agents. Bioorg. Med. Chem. 2014, 22, 5804-5812. [CrossRef] [PubMed]

11. Chugunova, E.; Boga, C.; Sazykin, I.; Cino, S.; Micheletti, G.; Mazzanti, A.; Sazykina, M.; Burilov, A.; Khmelevtsova, L.; Kostina, N. Synthesis and antimicrobial activity of novel structural hybrids of benzofuroxan and benzothiazole derivatives. Eur. J. Med. Chem. 2015, 93, 349-359. [CrossRef] [PubMed]

12. Gurdal, E.E.; Buclulgan, E.; Durmaz, I.; Cetin-Atalay, R.; Yarim, M. Synthesis and Anticancer Activity Evaluation of Some Benzothiazole-Piperazine Derivatives. Anticancer Agents Med. Chem. 2015, 15, 382-389. [CrossRef] [PubMed]

13. Puranik, N.V.; Puntambekar, H.M.; Srivastava, P. Antidiabetic potential and enzyme kinetics of benzothiazole derivatives and their non-bonded interactions with alpha-glucosidase and alpha-amylase. Med. Chem. Res. 2016, 25, 805-816. [CrossRef]

14. Taha, M.; Ismail, N.H.; Lalani, S.; Fatmi, M.Q.; Atia-tul-Wahab; Siddiqui, S.; Khan, K.M.; Imran, S.; Choudhary, M.I. Synthesis of novel inhibitors of alpha-glucosidase based on the benzothiazole skeleton containing benzohydrazide moiety and their molecular docking studies. Eur. J. Med. Chem. 2015, 92, 387-400. [CrossRef] [PubMed]

15. Agalave, S.G.; Maujan, S.R.; Pore, V.S. Click Chemistry: 1,2,3-Triazoles as Pharmacophores. Chem. Asian J. 2011, 6, 2696-2718. [CrossRef] [PubMed]

16. Hou, J.; Liu, X.; Shen, J.; Zhao, G.; Wang, P.G. The impact of click chemistry in medicinal chemistry. Expert Opin. Drug Discov. 2012, 7, 489-501. [CrossRef] [PubMed]

17. Saraei, M.; Ghasemi, Z.; Dehghan, G.; Hormati, M.; Ojaghi, K. Synthesis of some novel 1,2,3-triazole derivatives containing kojic acid moiety and evaluation for their antioxidant activity. Monatsh. Chem. 2017, 148, 917-923. [CrossRef]

18. Thomas, K.D.; Adhikari, A.V.; Shetty, N.S. Design, synthesis and antimicrobial activities of some new quinoline derivatives carrying 1,2,3-triazole moiety. Eur. J. Med. Chem. 2010, 45, 3803-3810. [CrossRef] [PubMed]

19. Boechat, N.; Ferreira, V.F.; Ferreira, S.B.; Ferreira, M.D.L.G.; da Silva, F.D.C.; Bastos, M.M.; Costa, M.D.S.; Lourenco, M.C.S.; Pinto, A.C.; Krettli, A.U.; et al. Novel 1,2,3-Triazole Derivatives for Use against Mycobacterium tuberculosis H37Rv (ATCC 27294) Strain. J. Med. Chem. 2011, 54, 5988-5999. [CrossRef] [PubMed]

20. Kamal, A.; Shankaraiah, N.; Devaiah, V.; Reddy, K.L.; Juvekar, A.; Sen, S.; Kurian, N.; Zingde, S. Synthesis of 1,2,3-triazole-linked pyrrolobenzodiazepine conjugates employing 'click' chemistry: DNA-binding affinity and anticancer activity. Bioorg. Med. Chem. Lett. 2008, 18, 1468-1473. [CrossRef] [PubMed]

21. Chinthala, Y.; Domatti, A.K.; Sarfaraz, A.; Singh, S.P.; Arigari, N.K.; Gupta, N.; Satya, S.K.V.N.; Kumar, J.K.; Khan, F.; Tiwari, A.K.; et al. Synthesis, biological evaluation and molecular modeling studies of some novel thiazolidinediones with triazole ring. Eur. J. Med. Chem. 2013, 70, 308-314. [CrossRef] [PubMed]

22. Chinthala, Y.; Thakur, S.; Tirunagari, S.; Chinde, S.; Domatti, A.K.; Arigari, N.K.; Srinivas, K.V.N.S.; Alam, S.; Jonnala, K.K.; Khan, F.; et al. Synthesis, docking and ADMET studies of novel chalcone triazoles for anti-cancer and anti-diabetic activity. Eur. J. Med. Chem. 2015, 93, 564-573. [CrossRef] [PubMed]

23. Jabeen, F.; Shehzadi, S.A.; Fatmi, M.Q.; Shaheen, S.; Iqbal, L.; Afza, N.; Panda, S.S.; Ansari, F.L. Synthesis, in vitro and computational studies of 1,4-disubstituted 1,2,3-triazoles as potential alpha-glucosidase inhibitors. Bioorg. Med. Chem. Lett. 2016, 26, 1029-1038. [CrossRef] [PubMed]

24. Wang, G.; Peng, Z.; Wang, J.; Li, J.; Li, X. Synthesis and biological evaluation of novel 2,4,5-triarylimidazole-1,2,3-triazole derivatives via click chemistry as $\alpha$-glucosidase inhibitors. Bioorg. Med. Chem. Lett. 2016, 26, 5719-5723. [CrossRef] [PubMed] 
25. Wang, G.; Peng, Z.; Wang, J.; Li, X.; Li, J. Synthesis, in vitro evaluation and molecular docking studies of novel triazine-triazole derivatives as potential $\alpha$-glucosidase inhibitors. Eur. J. Med. Chem. 2017, 125, 423-429. [CrossRef] [PubMed]

26. Wang, G.; Wang, J.; He, D.; Li, X.; Li, J.; Peng, Z. Synthesis and biological evaluation of novel 1,2,4-triazine derivatives bearing carbazole moiety as potent $\alpha$-glucosidase inhibitors. Bioorg. Med. Chem. Lett. 2016, 26, 2806-2809. [CrossRef] [PubMed]

27. Wang, G.; Chen, M.; Wang, J.; Peng, Y.; Li, L.; Xie, Z.; Deng, B.; Chen, S.; Li, W. Synthesis, biological evaluation and molecular docking studies of chromone hydrazone derivatives as $\alpha$-glucosidase inhibitors. Bioorg. Med. Chem. Lett. 2017, 27, 2957-2961. [CrossRef] [PubMed]

28. Wang, G.; Peng, Z.; Wang, J.; Li, J.; Li, X. Synthesis, biological evaluation and molecular docking study of $\mathrm{N}$-arylbenzo[d]oxazol-2-amines as potential $\alpha$-glucosidase inhibitors. Bioorg. Med. Chem. 2016, 24, 5374-5379. [CrossRef] [PubMed]

29. Christou, S.; Edwards, A.C.; Pritchard, R.G.; Quayle, P.; Song, Y.; Stratford, I.J.; Williams, K.F.; Whitehead, R.C. Synthesis of hybrid natural product analogues with anti-tumour properties. Tetrahedron 2016, 72, 5433-5443. [CrossRef]

Sample Availability: Samples of the compounds $6 a-6 s$ are available from the authors. 